\title{
Development and Validation of an UHPLC-QqQ-MS Technique for Simultaneous Determination of Ten Bioactive Components in Fangji Huangqi Tang
}

\author{
Xiaoli Wang, ${ }^{1,2,3}$ Xiao Liu, ${ }^{1,2,3}$ Tingting Zhu, ${ }^{1}$ and Baochang Cai ${ }^{1,2,3}$ \\ ${ }^{1}$ School of Pharmacy, Nanjing University of Chinese Medicine, Nanjing 210023, China \\ ${ }^{2}$ Engineering Center of State Ministry of Education for Standardization of Chinese Medicine Processing, Nanjing University of \\ Chinese Medicine, Nanjing 210023, China \\ ${ }^{3}$ Nanjing Haichang Chinese Medicine Group Corporation, Nanjing 210061, China
}

Correspondence should be addressed to Xiao Liu; liuxiao04_0@163.com and Baochang Cai; bccai@126.com

Received 3 March 2016; Revised 27 April 2016; Accepted 8 May 2016

Academic Editor: Vito Verardo

Copyright (C) 2016 Xiaoli Wang et al. This is an open access article distributed under the Creative Commons Attribution License, which permits unrestricted use, distribution, and reproduction in any medium, provided the original work is properly cited.

\begin{abstract}
The aim of this study is to develop an ultrahigh performance liquid chromatography method coupled with triple quadrupole mass spectrometry for simultaneous determination of tetrandrine, fangchinoline, atractylenolide I, atractylenolide III, calycosin7-O- $\beta$-D-glucoside, glycyrrhizin, liquiritin, isoliquiritin, liquiritigenin, and isoliquiritigenin in Fangji Huangqi Tang (FHT). The chromatographic separation was performed on a reversed- $\mathrm{C}_{18}$ column, eluted with a mixture of $0.1 \%$ acetic acid and acetonitrile at $0.4 \mathrm{~mL} / \mathrm{min}$. The separation of these ten compounds was achieved by linear gradient elution. The method was strictly validated with respect to specificity, precision, accuracy, and repeatability. All the compounds showed good linearities $(r \geq 0.999)$. The LOQs of the ten components were $0.36,0.18,0.09,0.43,0.02,1.89,0.26,0.18,0.61$, and $0.48 \mathrm{ng} / \mathrm{mL}$ for tetrandrine, fangchinoline, atractylenolide I, atractylenolide III, calycosin-7-O- $\beta$-D-glucoside, glycyrrhizin, liquiritin, isoliquiritin, liquiritigenin, and isoliquiritigenin, respectively. The LODs of the ten components were $0.11,0.05,0.03,0.13,0.01,0.57,0.08$, $0.05,0.18$, and $0.14 \mathrm{ng} / \mathrm{mL}$ for tetrandrine, fangchinoline, atractylenolide I, atractylenolide III, calycosin-7-O- $\beta$-D-glucoside, glycyrrhizin, liquiritin, isoliquiritin, liquiritigenin, and isoliquiritigenin, respectively. The method was proven to be specific and reliable, which would provide a meaningful basis for the quality control and evaluation of FHT during its clinical application.
\end{abstract}

\section{Introduction}

Fangji Huangqi Tang (FHT) is a classical formula of traditional Chinese medicine (TCM). It was firstly recorded in "Jing Gui Yao Lue" by Zhongjing Zhang in the Han Dynasty. The formula consisted of four herbs, including Stephania tetrandra S. Moore, Astragalus membranaceus (Fisch.) Bge., Atractylodes macrocephala Koidz., and Glycyrrhiza uralensis Fisch. Lots of doctors recommended FHT for the treatment of chronic glomerulonephritis, cardiac edema, and rheumatic arthritis in Chinese clinical application based on their actual experience [1]. Also, experts began to do their research work to reveal the potential effective compounds in FHT [2]. For safe and effective use of FHT in the clinic, quality control and evaluation of this formula with multiple compounds' identification and determination became essential and important. However, to our knowledge, there are no relative reports until now. It has already been found out that FHT contains different kinds of components, such as flavonoids (liquiritigenin, isoliquiritigenin, liquiritin, formononetin, and calycosin), alkaloids (fangchinoline and tetrandrine), lactones (atractylenolide I, atractylenolide II, atractylenolide III, and atractylon), and saponins (astragaloside I, astragaloside II, astragaloside III, and astragaloside IV) [3]. Among these compositions, fangchinoline, tetrandrine, and liquiritigenin, as well as calycosin, all showed good effects on anti-inflammatory and improving immunity [47]. The critical strategy for quality control and evaluation of FHT should be applied to study as much of these effective components as possible. 
Several common analytical techniques were usually used for multiple chemical compounds' determination in TCM, including HPLC-DAD, UV, HPLC-ELSD, and LC-ICPMS [8-14]. However, these methods showed lots of drawbacks and technical shortcomings, such as time-consuming sample running, poor sensitivity, peak interferences, and limitation of detectors on components identification. As it is widely accepted, the UHPLC-QqQ-MS technique has a special advantage of high resolution and acute sensitivity. And it is now a very powerful tool for the determination of complex chemical compounds in TCM prescriptions, by which the identification of each analyte is also accomplished at the same time. For this reason, a simple, rapid, and sensitive method for simultaneous determination of 10 components in FHT was developed based on UHPLC-QqQ-MS technique in this paper, and the feasibility of this technique on TCM quality control and evaluation was strongly demonstrated.

\section{Experimental}

2.1. Reagents and Materials. Astragalus membranaceus (Fisch.) Bge. and Glycyrrhiza uralensis Fisch. were obtained from Nanjing Haichang Chinese Medicine Group Corporation (Nanjing, China). Stephania tetrandra S. Moore and Atractylodes macrocephala Koidz. were obtained from Hebei Chinese Medicine Group Corporation (Hebei, China). Fangchinoline, tetrandrine, calycosin-7-O- $\beta$-D-glucoside, atractylenolide I, atractylenolide III, liquiritin, isoliquiritin, liquiritigenin, isoliquiritigenin, and glycyrrhizin with purity of $99 \%$ or higher were purchased from the Sichuan Victor Biological Technology Co. (Chengdu, China). HPLC-grade methanol and acetonitrile were purchased from E. Merck (Merck, Darmstadt, Germany). Purified water was from a Milli-Q system (Millipore Corporation, Bedford, MA, USA). Formic acid was of analytical grade and was obtained from Nanjing Chemical Reagent Company (Nanjing, China).

2.2. Instruments. The UHPLC system (Shimadzu, Kyoto, Japan) consisted of LC-30AD binary pump, an autosampler (Model SIL-30SD), an online degasser (DGU-20A5R), and a column temperature controller compartment (CTO-30A). An Extend- $\mathrm{C}_{18}$ column (Agilent, USA, $2.1 \mathrm{~mm} \times 100 \mathrm{~mm}$, $1.8 \mu \mathrm{m})$ at a temperature of $30^{\circ} \mathrm{C}$ was used for separation. Mass spectrometric detection was performed using triple quadrupole 5500 instrument (AB Sciex, Concord, Ontario, Canada) equipped with an electrospray ionization (ESI) source.

2.3. UHPLC-MS/MS Conditions. The separation of these ten compounds was achieved by linear gradient elution using a mobile phase consisting of $0.1 \%(\mathrm{v} / \mathrm{v})$ aqueous formic acid (A) and acetonitrile (B). The gradient program was as follows: $0.1-0.8 \mathrm{~min}, 5 \% \mathrm{~B}-50 \% \mathrm{~B} ; 0.8-3 \mathrm{~min}, 50 \% \mathrm{~B}-90 \% \mathrm{~B} ; 3-$ $3.1 \mathrm{~min}, 90 \% \mathrm{~B}-5 \% \mathrm{~B}$. The flow rate was set at $0.4 \mathrm{~mL} / \mathrm{min}$. The samples were placed in an autosampler at $4^{\circ} \mathrm{C}$ prior to injection and the injection volume was $10 \mu \mathrm{L}$. Both positive and negative ionization mass spectrometric analyses were conducted due to the physicochemical properties of the ten analytes. The scan mode was set as multiple reactions monitoring (MRM) and the selected monitor ions were $\mathrm{m} / z$ 623.2/174.1 for tetrandrine, $m / z 609.3 / 367.1$ for fangchinoline, $m / z$ 233.1/187.2 for atractylenolide I, $m / z$ 249.1/231.0 for atractylenolide III, $m / z \quad 447.2 / 285.1$ for calycosin-7-O- $\beta$-Dglucoside, $m / z$ 821.3/351.2 for glycyrrhizin, $m / z 417.1 / 255.1$ for liquiritin, $m / z 417.1 / 255.1$ for isoliquiritin, $m / z 256.2 / 135.0$ for liquiritigenin, and $\mathrm{m} / z$ 256.2/135.0 for isoliquiritigenin. Also, another selected monitor ion pair was selected for the identification purpose for each analyte. They were $\mathrm{m} / \mathrm{z}$ 623.2/181.3 for tetrandrine, $m / z 609.3 / 192.2$ for fangchinoline, $m / z$ 233.1/215.1 for atractylenolide I, $m / z$ 249.1/163.0 for atractylenolide III, $m / z \quad 447.2 / 270.0$ for calycosin-7-O- $\beta$-Dglucoside, $m / z$ 821.3/193.1 for glycyrrhizin, $m / z$ 417.1/135.0 for liquiritin, $m / z 417.1 / 135.0$ for isoliquiritin, $m / z$ 256.2/119.1 for liquiritigenin, and $m / z 256.2 / 119.1$ for isoliquiritigenin. Source condition parameters were as follows: curtain gas set at 35 , ion source temperature adjusted to $550^{\circ} \mathrm{C}$, and ion source gas 1 (GS 1) and ion source gas 2 (GS 2) both at 55.

2.4. Preparation of FHT Sample. According to the original composition and preparation process of FHT recorded in "Jin-Gui-Yao-Lue," FHT was obtained in the following procedure: pieces of Stephania tetrandra S. Moore (12 g), Astragalus membranaceus (Fisch.) Bge. (15 g), Atractylodes macrocephala Koidz. (9 g), and Glycyrrhiza uralensis Fisch. $(6 \mathrm{~g})$ were mixed together in a ratio $(4: 5: 3: 2, \mathrm{w} / \mathrm{w} / \mathrm{w} / \mathrm{w})$ and macerated in deionized water for $30 \mathrm{~min}$ before being decocted twice with boiling water (1:10, w/v and $1: 8, \mathrm{w} / \mathrm{v}$, resp.), $20 \mathrm{~min}$ for each time. The solution was then filtered through a four-layer mesh and was diluted with water to $1000 \mathrm{~mL} .2 \mathrm{~mL}$ of the above solution was added to $50 \mathrm{~mL}$ vessel, and $15 \mathrm{~mL}$ of $60 \%$ methanol solution was added for ultrasonic extraction. Then, moderate volume of $60 \%$ methanol solution was added to the scale of the $50 \mathrm{~mL}$ vessel. The solution was centrifuged at $12,000 \mathrm{rpm}$ for $5 \mathrm{~min}$ and was filtered through $0.22 \mu \mathrm{m}$ membrane. The filtrate collected was then used for UHPLCQqQ-MS analysis.

\subsection{Calibration Curves and Limits of Detection and Quan-} tification. Stock solutions of the 10 analytes were separately prepared by diluting the reference standards with methanol. The chemical structures of the compounds were shown in Figure 1 . All the stock solutions were separately kept at $4^{\circ} \mathrm{C}$. A mixed working solution was prepared before use every time. All the standards were soluble in methanol and completely dissolved in the mixed working solution. And the mixed working solution containing 10 compounds was diluted to appropriate concentration ranges for the construction of calibration curves. The calibration curve of each compound was performed with at least seven appropriate concentrations levels. The concentrations of the 10 components in Fangji Huangqi Tang should be included in the range of the standard curve. The limit of detection (LOD) and limit of quantification (LOQ) under the present chromatographic conditions were determined at a signal-to-noise $(\mathrm{S} / \mathrm{N})$ ratio of 3 and 10 , respectively. 
<smiles>C=C1CCC[C@]2(C)C=C3OC(=O)C(C)=C3C[C@H]12</smiles>

Atractylenolide I

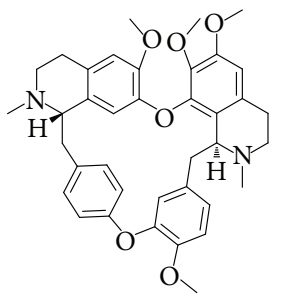

Tetrandrine<smiles>C=C1CCC[C@]2(C)C[C@]3(O)OC(=O)C(C)=C3CC12</smiles>

Atractylenolide III<smiles>COc1ccc(CN2CCc3cc(OC)c(O)c(Oc4ccc5c(c4)CN(C)CC5)c32)cc1Cc1ccc(OC)c(OC)c1</smiles>

Fangchinoline<smiles>COc1ccc(-c2coc3cc(O[C@@H]4O[C@H](CO)[C@@H](O)[C@H](O)[C@H]4O)ccc3c2=O)cc1O</smiles>

Calycosin-7-O- $\beta$-D-glucoside<smiles>O=C1CC(c2ccc(OC3OC(CO)C(O)C(O)C3O)cc2)Oc2cc(O)ccc21</smiles><smiles>[H]</smiles><smiles>CCC(C)CCCOC1OC(O)C(C(=O)O)C(O)C1O</smiles>

Liquiritin

Glycyrrhizin<smiles>O=C1C[C@H](c2ccc(O)cc2)Oc2cc(O)ccc21</smiles>

Liquiritigenin<smiles>CCCCOc1ccc(C(=O)C=Cc2ccc(O)cc2)c(O)c1</smiles>

Isoliquiritigenin<smiles>O=C(/C=C/c1ccc(OC2OC(CO)[C@H](O)C(O)[C@H]2O)cc1)c1ccc(O)cc1O</smiles>

Isoliquiritin

FIGURE 1: The chemical structures of the ten selected major compounds of FHT.

2.6. Precision, Accuracy, and Stability. Intraday variations within one day were all taken into consideration to determine the precision of the developed method. The relative standard deviation (RSD) was taken as a measurement of repeatability. A recovery test was utilized to evaluate the accuracy of this method. Certain amounts of 10 standards were accurately added to a FHT sample and then processed and analyzed as described in Sections 2.2 and 2.3. The total amount of each analyte was calculated from the corresponding calibration curve, and the recovery of each analyte was calculated by the following equation: recovery $(\%)=$ amount $_{\text {determined }}-$

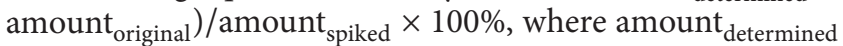
is the determined total amount of each analyte, amount ${ }_{\text {original }}$ is the original amount of each analyte in samples determined,

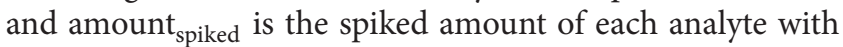
$80 \%, 100 \%$, and $120 \%$ ratio of the original amount. In order to investigate the stability of the processed sample solution, the same sample solution was stored at $4^{\circ} \mathrm{C}$ and analyzed every $12 \mathrm{~h}$ for 1 day. In addition, the 10 stock solutions were all determined every week to investigate whether obvious degradation had happened under their preservation condition.

\section{Results and Discussion}

3.1. Optimization of Sample Preparation and Experimental Conditions. According to the published papers, many different kinds of components were identified in FHT including
TABLE 1: List of selected MRM parameters, including CE, DP, EP, and CXP for the 10 major compounds of Fangji Huangqi Tang.

\begin{tabular}{lcccc}
\hline Compound & CE & DP & EP & CXP \\
\hline Tetrandrine & 53.90 & 39.86 & 3.93 & 10.83 \\
Fangchinoline & 49.43 & 27.13 & 4.29 & 27.3 \\
Atractylenolide I & 22.61 & 63.96 & 6.81 & 14.00 \\
Atractylenolide III & 14.42 & 54.80 & 3.14 & 14.00 \\
Calycosin-7-O- $\beta$-D-glucoside & 16.11 & 57.00 & 6.38 & 14.00 \\
Glycyrrhizin & -52.15 & -14.67 & -13.72 & -22.52 \\
Liquiritin & -23.39 & -17.32 & -10.15 & -21.88 \\
Isoliquiritin & -23.39 & -17.32 & -10.15 & -21.88 \\
Liquiritigenin & -21.65 & -65.84 & -9.14 & -17.00 \\
Isoliquiritigenin & -21.65 & -65.84 & -9.14 & -17.00 \\
\hline
\end{tabular}

alkaloids, flavonoid, and lactones. Also, these chemical compounds were proved to be the main effective components in FHT. The ten selected compounds for simultaneous determination belonged to different chemical classes and covered a wide polarity range. So, it is of great significance to screen out an appropriate sample preparation method based on the effective extraction for various effective compounds. Therefore, a novel sample method has been developed to collect different polar components on a solid phase extraction column. This simple and convenient technique was suitable 


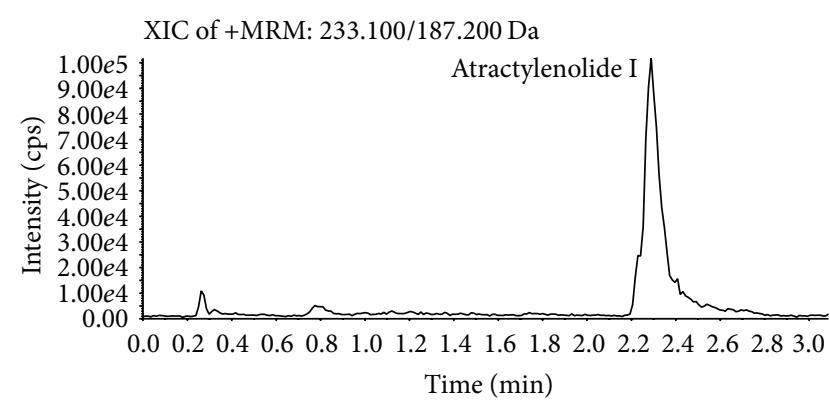

(a)

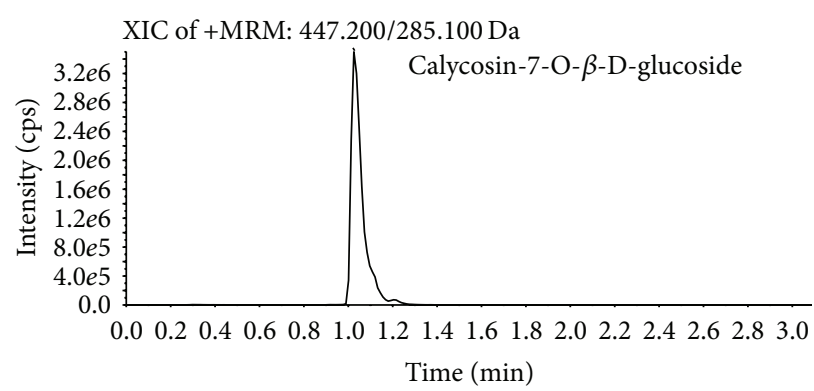

(c)

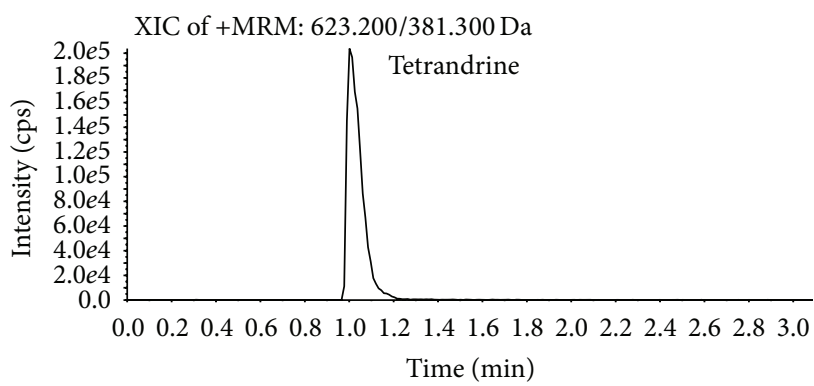

(e)

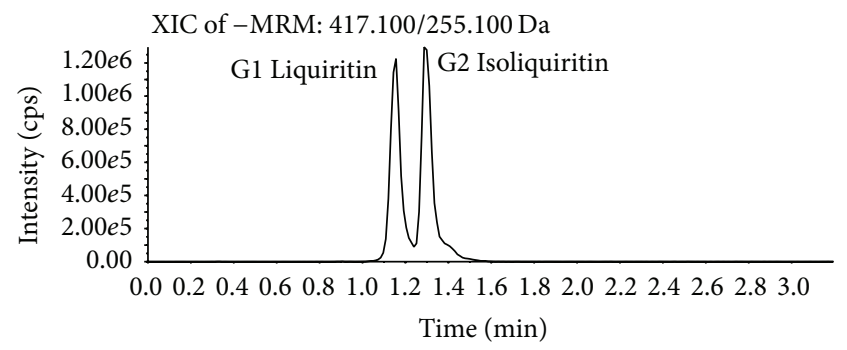

(g)

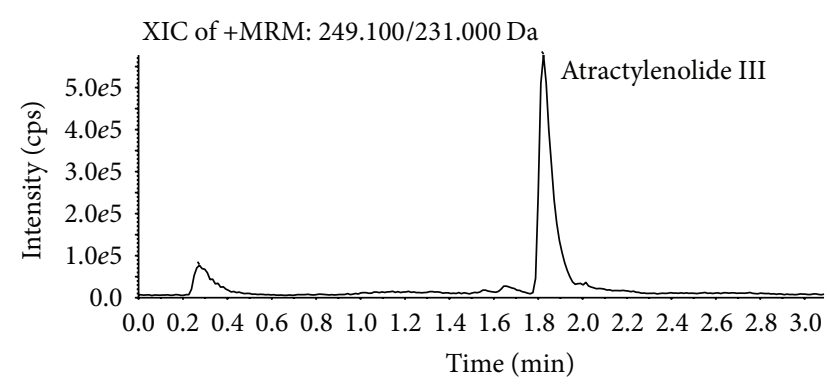

(b)

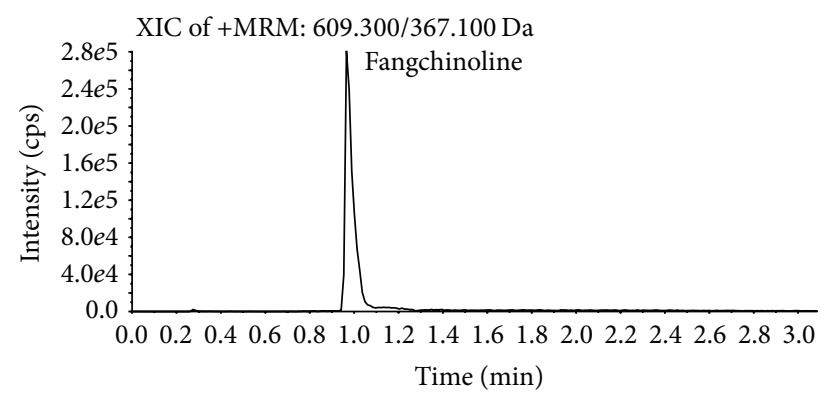

(d)

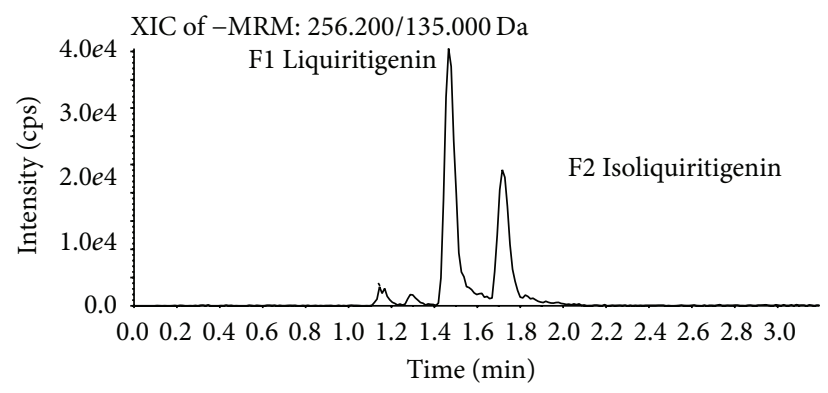

(f)

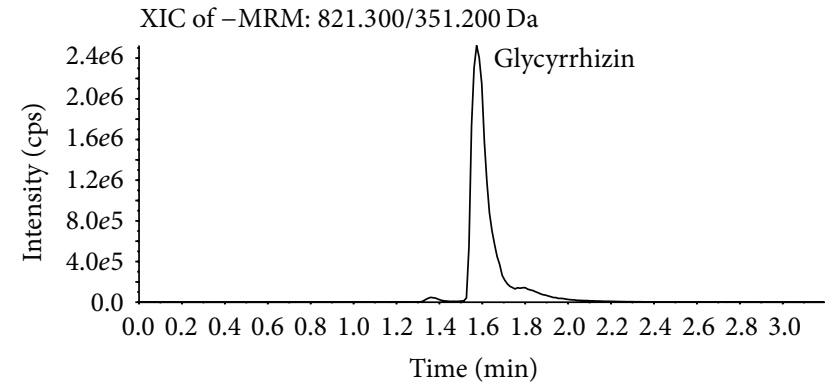

(h)

Figure 2: Representative extract ions chromatograms for the determination of the 10 analytes. (a) Atractylenolide I (2.28 min). (b) Atractylenolide III (1.83 min). (c) Calycosin-7-O- $\beta$-D-glucoside (1.04 min). (d) Fangchinoline (0.98 min). (e) Tetrandrine (1.01 min). (f) Liquiritigenin (1.47 $\mathrm{min}$ ) and isoliquiritigenin $(1.72 \mathrm{~min})$. (g) Liquiritin (1.17 min) and isoliquiritin (1.31 min). (h) Glycyrrhizin (1.60 min).

for traditional Chinese medicine prescriptions detection by enhancing the sample polarity scope, as well as reducing the pollution of the instrument.

It was difficult for researchers to perform good separation for the chemical constituents in FHT because of the complexity of the composition and the existence of isomers.
In this paper, the UHPLC conditions were well optimized, including the type of column, column temperature, mobile phase system, and flow rate. After a comparison of different brands of columns including Agilent $\mathrm{C}_{18}$, Waters $\mathrm{C}_{18}$, and Thermo $\mathrm{C}_{18}$, Agilent column finally gave out sound separation for the ten compounds to be determined. In 
TABLE 2: Calibration curves, LOD, and LOQ of the 10 major compounds.

\begin{tabular}{|c|c|c|c|c|c|c|c|}
\hline Compounds & Calibration curve & $r$ & $\begin{array}{l}\text { Linear } \\
\text { range } \\
(\mathrm{ng} / \mathrm{mL})\end{array}$ & $\begin{array}{c}\text { LOQ } \\
(\mathrm{ng} / \mathrm{mL})\end{array}$ & $\begin{array}{c}\text { LOD } \\
(\mathrm{ng} / \mathrm{mL})\end{array}$ & $\begin{array}{c}\mathrm{MS} / \mathrm{MS} \\
(m / z)\end{array}$ & Detected ion \\
\hline Tetrandrine & $y=28104.45 x+2073.02$ & 0.9996 & $1.63-104.16$ & 0.36 & 0.11 & $623.2 / 381.3$ & {$[\mathrm{M}+\mathrm{H}]^{+}$} \\
\hline Fangchinoline & $y=23500.46 x+2211.84$ & 0.9991 & $1.84-117.54$ & 0.18 & 0.05 & $609.3 / 367.1$ & {$[\mathrm{M}+\mathrm{H}]^{+}$} \\
\hline Atractylenolide I & $y=116476 x+37509.7$ & 0.9998 & $0.37-23.55$ & 0.09 & 0.03 & $233.1 / 187.2$ & {$[\mathrm{M}+\mathrm{H}]^{+}$} \\
\hline $\begin{array}{l}\text { Atractylenolide } \\
\text { III }\end{array}$ & $y=114683 x+86369.7$ & 0.9993 & $0.56-35.63$ & 0.43 & 0.13 & 249.1/231.0 & {$[\mathrm{M}+\mathrm{H}]^{+}$} \\
\hline $\begin{array}{l}\text { Calycosin-7-O- } \beta \text { - } \\
\text { D-glucoside }\end{array}$ & $y=835469 x+982159$ & 0.9999 & $9.94-635.92$ & 0.02 & 0.01 & $447.2 / 285.1$ & {$[\mathrm{M}+\mathrm{H}]^{+}$} \\
\hline Glycyrrhizin & $y=8268.92 x+177769$ & 0.9991 & $\begin{array}{c}31.40- \\
2009.56\end{array}$ & 1.89 & 0.57 & $821.3 / 351.2$ & {$[\mathrm{M}-\mathrm{H}]^{-}$} \\
\hline Liquiritin & $y=9740.06 x+3971180$ & 0.9996 & $\begin{array}{l}123.75- \\
7920.00\end{array}$ & 0.26 & 0.08 & $417.1 / 255.1$ & {$[\mathrm{M}-\mathrm{H}]^{-}$} \\
\hline Isoliquiritin & $y=20012.34 x+4672350$ & 0.9991 & $\begin{array}{c}30.38- \\
1944.03\end{array}$ & 0.18 & 0.05 & $417.1 / 255.1$ & {$[\mathrm{M}-\mathrm{H}]^{-}$} \\
\hline Liquiritigenin & $y=16315.93 x+140.37$ & 0.9995 & $2.12-135.55$ & 0.61 & 0.18 & $256.2 / 135.0$ & {$[\mathrm{M}-\mathrm{H}]^{-}$} \\
\hline Isoliquiritigenin & $y=35718.6 x+2842.59$ & 0.9998 & $0.61-39.01$ & 0.48 & 0.14 & $256.2 / 135.0$ & {$[\mathrm{M}-\mathrm{H}]^{-}$} \\
\hline
\end{tabular}

TABle 3: Precision and repeatability for the assay of the 10 major compounds.

\begin{tabular}{|c|c|c|c|c|c|}
\hline \multirow{2}{*}{ Compounds } & \multirow{2}{*}{ Concentration $(\mathrm{ng} / \mathrm{mL})$} & \multicolumn{2}{|c|}{ Precision $(n=6)$} & \multicolumn{2}{|c|}{ Repeatability $(n=5)$} \\
\hline & & Found & RSD (\%) & Found & RSD (\%) \\
\hline Tetrandrine & 52.08 & $51.49 \pm 0.46$ & 0.89 & $53.52 \pm 1.16$ & 2.15 \\
\hline Fangchinoline & 58.77 & $57.56 \pm 0.49$ & 0.84 & $55.87 \pm 0.85$ & 1.52 \\
\hline Atractylenolide I & 11.77 & $11.51 \pm 0.26$ & 2.27 & $12.26 \pm 0.26$ & 2.15 \\
\hline Atractylenolide III & 17.82 & $17.47 \pm 0.14$ & 0.78 & $21.60 \pm 0.58$ & 2.68 \\
\hline Calycosin-7-O- $\beta$-D-glucoside & 317.96 & $317.81 \pm 0.58$ & 0.18 & $145.27 \pm 1.86$ & 1.28 \\
\hline Glycyrrhizin & 1003.78 & $1003.86 \pm 1.18$ & 0.12 & $1661.02 \pm 34.97$ & 2.11 \\
\hline Liquiritin & 3960.00 & $3941.00 \pm 46.23$ & 1.17 & $3303.74 \pm 24.75$ & 0.75 \\
\hline Isoliquiritin & 972.02 & $974.07 \pm 1.20$ & 0.12 & $695.73 \pm 8.31$ & 1.19 \\
\hline Liquiritigenin & 67.78 & $68.90 \pm 0.89$ & 1.29 & $44.50 \pm 0.83$ & 1.86 \\
\hline Isoliquiritigenin & 19.51 & $18.94 \pm 0.48$ & 2.53 & $3.11 \pm 0.06$ & 2.03 \\
\hline
\end{tabular}

addition, different kinds of mobile phase systems such as acetonitrile and methanol with a variety of modifiers (including formic acid and acetic acid) were tested. As a result, a solution of $0.1 \%(\mathrm{v} / \mathrm{v})$ aqueous formic acid and acetonitrile at $0.4 \mathrm{~mL} / \mathrm{min}$ was used as the optimal chromatographic elution system.

Typical source conditions were used as follows: curtain gas was set at 35 ; ion source temperature was adjusted to $500^{\circ} \mathrm{C}$; ion source gas 1 (GS 1) and ion source gas 2 (GS 2) were both set at 55 . Independent parameters for the detection of each compound were optimized by injecting standard solution into MS system under MRM mode, including the declustering potential (DP), the collision energy (CE), the entrance potential (EP), and the collision cell exit potential (CXP). These parameters for the detection of the ten constituents were shown in Table 1.

3.2. Method Validation. Chromatographic peaks of FHT were identified by comparing the retention time with that of each reference compound. The representative extract ions chromatograms of the standard mixture solution were shown in Figure 2. (a) stands for fangchinoline (0.98 min); (b) stands for atractylenolide I $(2.28 \mathrm{~min})$; (c) stands for tetrandrine (1.01 min); (d) stands for atractylenolide III (1.83 min); (e) stands for calycosin-7-O- $\beta$-D-glucoside (1.04 min); (f) stands for liquiritigenin (1.47 $\mathrm{min}$ ) and isoliquiritigenin (1.72 $\mathrm{min})$; (g) stands for liquiritin (1.17 $\mathrm{min}$ ) and isoliquiritin (1.31 $\mathrm{min})$; (h) stands for glycyrrhizin $(1.60 \mathrm{~min})$. According to the massto-electric charge ratio of each compound, all compounds were detected in different channels without interfering with each other. Table 2 shows the regression equation for each reference compound, as well as the LOD and LOQ values and the mass spectrometry information. All the components showed good linearity $(r>0.9991)$ in a relatively wide concentration range. Tables 3 and 4 provide precision and recovery results of the 10 components, respectively. The intraday variations of the compounds were $<2.53 \%$, the relative standard deviation (RSD) of repeatability test was below $2.68 \%$ for all the analytes of interest, and the extraction recoveries of the components ranged from $98.51 \%$ to $101.10 \%$. 
TABLE 4: Recovery of the 10 major compounds in Fangji Huangqi Tang $(n=3)$.

\begin{tabular}{|c|c|c|c|c|c|}
\hline Compounds & Original amount (ng) & Spiked amount (ng) & Detected amount (ng) & Recovery (\%) & RSD (\%) \\
\hline \multirow{3}{*}{ Tetrandrine } & \multirow{3}{*}{1304.50} & 1045.00 & $2343.50 \pm 2.50$ & \multirow{3}{*}{99.24} & \multirow{3}{*}{0.91} \\
\hline & & 1306.00 & $2601.00 \pm 20.50$ & & \\
\hline & & 1567.50 & $2857.00 \pm 18.00$ & & \\
\hline \multirow{3}{*}{ Fangchinoline } & \multirow{3}{*}{1392.50} & 1111.50 & $2480.00 \pm 27.50$ & \multirow{3}{*}{99.27} & \multirow{3}{*}{1.08} \\
\hline & & 1389.50 & $2782.50 \pm 5.00$ & & \\
\hline & & 1667.00 & $3057.00 \pm 21.50$ & & \\
\hline \multirow{3}{*}{ Atractylenolide I } & \multirow{3}{*}{294.00} & 232.00 & $529.00 \pm 2.50$ & \multirow{3}{*}{101.1} & \multirow{3}{*}{1.40} \\
\hline & & 290.00 & $584.00 \pm 5.50$ & & \\
\hline & & 348.00 & $647.50 \pm 0.50$ & & \\
\hline \multirow{3}{*}{ Atractylenolide III } & \multirow{3}{*}{540.00} & 431.00 & $965.50 \pm 10.00$ & \multirow{3}{*}{99.88} & \multirow{3}{*}{1.78} \\
\hline & & 538.50 & $1079.00 \pm 1.50$ & & \\
\hline & & 646.50 & $1192.00 \pm 19.50$ & & \\
\hline \multirow{3}{*}{ Calycosin-7-O- $\beta$-D-glucoside } & \multirow{3}{*}{3622.00} & 2897.00 & $6512.50 \pm 6.00$ & \multirow{3}{*}{99.59} & \multirow{3}{*}{0.29} \\
\hline & & 3621.00 & $7203.00 \pm 14.50$ & & \\
\hline & & 4345.50 & $7973.00 \pm 79.00$ & & \\
\hline \multirow{3}{*}{ Glycyrrhizin } & \multirow{3}{*}{41970.00} & 33575.00 & $75216.00 \pm 145.00$ & \multirow{3}{*}{98.74} & \multirow{3}{*}{0.78} \\
\hline & & 41969.00 & $83537.50 \pm 362.00$ & & \\
\hline & & 50362.50 & $91399.50 \pm 501.00$ & & \\
\hline \multirow{3}{*}{ Liquiritin } & \multirow{3}{*}{82593.50} & 66074.00 & $148436.50 \pm 254.50$ & \multirow{3}{*}{99.18} & \multirow{3}{*}{0.96} \\
\hline & & 82592.50 & $163987.50 \pm 1503.50$ & & \\
\hline & & 99111.00 & $181061.00 \pm 627.50$ & & \\
\hline \multirow{3}{*}{ Isoliquiritin } & \multirow{3}{*}{17393.50} & 13914.50 & $31167.50 \pm 95.50$ & \multirow{3}{*}{98.84} & \\
\hline & & 17393.00 & $34595.00 \pm 80.00$ & & 0.47 \\
\hline & & 20871.50 & $37982.00 \pm 52.00$ & & \\
\hline & & 890.50 & $1991.00 \pm 9.50$ & & \\
\hline Liquiritigenin & 1112.50 & 1113.00 & $2209.00 \pm 14.50$ & 98.51 & 1.18 \\
\hline & & 1335.50 & $2426.00 \pm 18.00$ & & \\
\hline & & 63.00 & $141.50 \pm 1.00$ & & \\
\hline Isoliquiritigenin & 79.00 & 78.50 & $156.50 \pm 0.50$ & 99.55 & 1.09 \\
\hline & & 94.50 & $173.50 \pm 0.50$ & & \\
\hline
\end{tabular}

Stability was evaluated by analyzing all the analytes in samples kept at $4^{\circ} \mathrm{C}$ for $24 \mathrm{~h}$. They were all found to be stable within $24 \mathrm{~h}(\mathrm{RSD}<3 \%)$. As a result, the developed method was proved to be precise, accurate, and sensitive for the simultaneous quantification of the major compounds of FHT.

3.3. Sample Analysis. The developed UHPLC-MS/MS method was subsequently applied to analysis and quality evaluation of FHT. The content of these major constituents in FHT was shown in Table 5.

\section{Conclusions}

A rapid UHPLC-MS/MS method was established for the comprehensive analysis of FHT. This method could separate the complex constituents in a short run time. Furthermore, MS/MS proposed high sensitivity which is helpful for the quantification of the low-content compounds. The method was successfully applied to the simultaneous determination of 10 bioactive compounds in FHT. This available, rapid, and reliable method is fit for routine analysis and effective quality control of this Chinese herbal prescription.

\section{Abbreviations}

UHPLC-QqQ-MS: Ultrahigh performance liquid chromatography method coupled with triple quadrupole mass spectrometry

TCM: $\quad$ Traditional Chinese medicine HPLC-DAD: $\quad$ High performance liquid chromatography with diode array detection

UV: Ultraviolet

HPLC-ELSD: High performance liquid chromatography with evaporative light scattering detector

LC-ICPMS: $\quad$ Liquid chromatography with inductively coupled plasma mass spectrometry. 


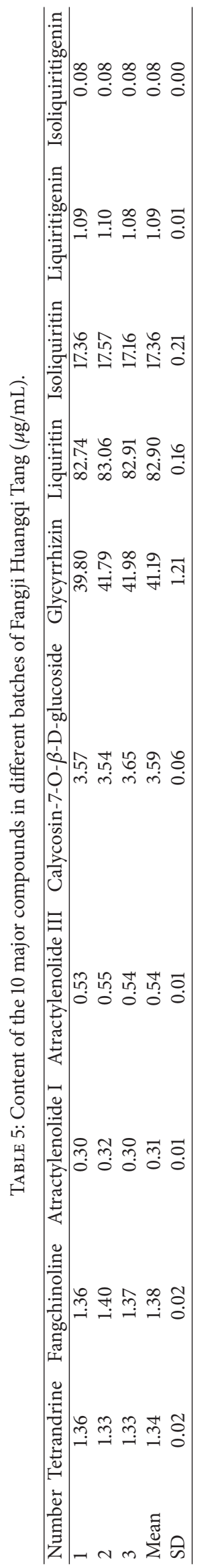




\section{Competing Interests}

The authors declare no competing interests regarding the publication of this paper.

\section{Acknowledgments}

This research was financially supported by the Youth Natural Science Foundation of Nanjing University of Chinese Medicine (no. 13XZR25), Natural Science Foundation of Jiangsu province (BK20140964), a project funded by the Priority Academic Program Development of Jiangsu Higher Education Institutions, and the National Natural Science Foundation of China (no. 81503207).

\section{References}

[1] G. Chen, H. Liu, and F. Cheng, "Fangjihuangqi Tang improved lower urinary tract dysfunction in benign prostatic hyperplasia rats model," Journal of Traditional Chinese Medicine, vol. 33, no. 3, pp. 349-354, 2013.

[2] X. Wang, X. Liu, X. Xu et al., "Screening and identification of multiple constituents and their metabolites of Fangji Huangqi Tang in rats by ultra-high performance liquid chromatography coupled with quadrupole time-of-flight tandem mass spectrometry basing on coupling data processing techniques," Journal of Chromatography B: Analytical Technologies in the Biomedical and Life Sciences, vol. 985, no. 15, pp. 14-28, 2015.

[3] X. L. Wang, X. Liu, H. Cai et al., "Ultra high performance liquid chromatography with tandem mass spectrometry method for the determination of tetrandrine and fangchinoline in rat plasma after oral administration of Fangji Huangqi Tang and Stephania tetrandraS. Moore extracts," Journal of Separation Science, vol. 38, no. 8, pp. 1286-1293, 2015.

[4] Y. C. Shen, C. J. Chou, W. F. Chiou, and C. F. Chen, "Antiinflammatory effects of the partially purified extract of radix Stephaniae tetrandrae: comparative studies of its active principles tetrandrine and fangchinoline on human polymorphonuclear leukocyte functions," Molecular Pharmacology, vol. 60, no. 5, pp. 1083-1090, 2001.

[5] H. S. Choi, H. S. Kim, K. R. Min et al., "Anti-inflammatory effects of fangchinoline and tetrandrine," Journal of Ethnopharmacology, vol. 69, no. 2, pp. 173-179, 2000.

[6] Y. W. Kim, R. J. Zhao, S. J. Park et al., "Anti-inflammatory effects of liquiritigenin as a consequence of the inhibition of NF- $\kappa \mathrm{B}-$ dependent iNOS and proinflammatory cytokines production," British Journal of Pharmacology, vol. 154, no. 1, pp. 165-173, 2008.

[7] W. Li, Y. N. Sun, X. T. Yan et al., "Flavonoids from Astragalus membranaceus and their inhibitory effects on LPS-stimulated pro-inflammatory cytokine production in bone marrowderived dendritic cells," Archives of Pharmacal Research, vol. 37, no. 2, pp. 186-192, 2014.

[8] B. Xie, T. Gong, M. Tang et al., "An approach based on HPLCfingerprint and chemometrics to quality consistency evaluation of Liuwei Dihuang Pills produced by different manufacturers," Journal of Pharmaceutical and Biomedical Analysis, vol. 48, no. 4, pp. 1261-1266, 2008.

[9] Z. Weng, C. Yan, Y. Wu, B. Cai, W. Li, and Z. Chen, "Determination of ten components in eucommia ulmoides oliver by high-performance liquid chromatography with dual detectors," Analytical Letters, vol. 47, no. 12, pp. 1978-1986, 2014.

[10] W. J. Kong, Y. L. Zhao, L. M. Shan, X. H. Xiao, and W. Y. Guo, "Investigation on the spectrum-effect relationships of EtOAc extract from Radix Isatidis based on HPLC fingerprints and microcalorimetry," Journal of Chromatography B, vol. 871, no. 1, pp. 109-114, 2008.

[11] Y. Jin, X. Xue, H. Shi, Y. Xiao, F. Zhang, and X. Liang, "HPLC and UPLC Switch for TCM analysis," World Science and Technology, vol. 10, no. 1, pp. 80-84, 2008.

[12] J. Zhang, Y. Liu, A. Jaquins-Gerstl, Z. Shu, A. C. Michael, and S. G. Weber, "Optimization for speed and sensitivity in capillary high performance liquid chromatography. The importance of column diameter in online monitoring of serotonin by microdialysis," Journal of Chromatography A, vol. 1251, pp. 54-62, 2012.

[13] N. Shraim, R. Clinckers, S. Sarre, Y. Michotte, and A. V. Eeckhaut, "Determination of reboxetine in rat brain microdialysates and plasma samples using liquid chromatography coupled to fluorescence detection," Journal of Chromatography B, vol. 898, pp. 53-61, 2012.

[14] H. Guo, H. Li, X. Liu, H. Cai, L. Wu, and B.-C. Cai, "Development and validation of a HPLC-UV-ESI-MS method for the simultaneous quantitation of ten bioactive compounds in Dahuang Fuzi Tang," Chinese Journal of Natural Medicines, vol. 12, no. 12, pp. 952-960, 2014. 

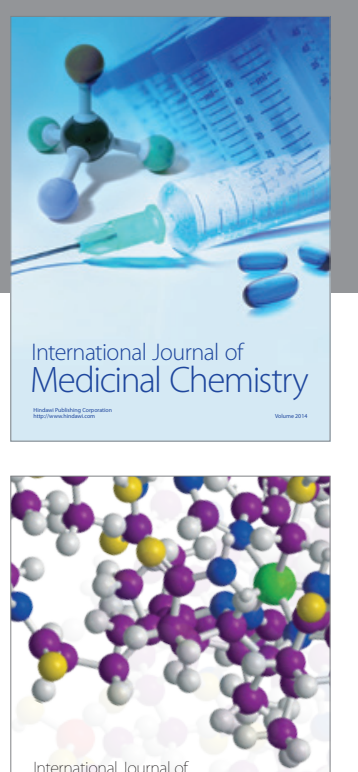

Carbohydrate Chemistry

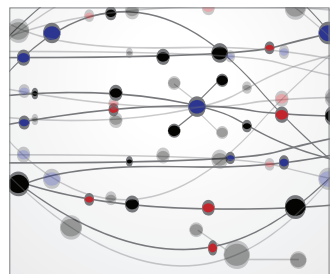

The Scientific World Journal
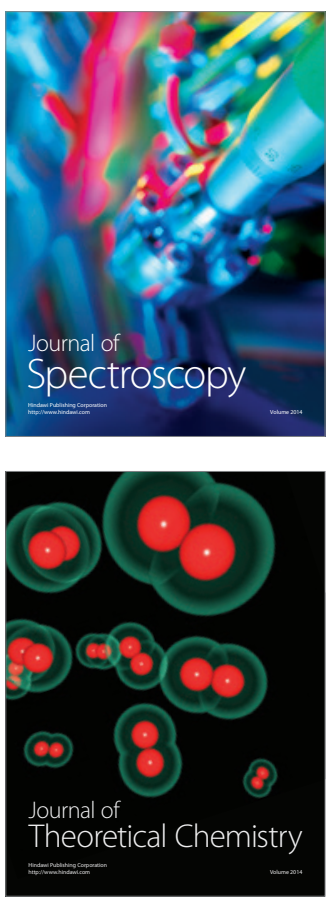
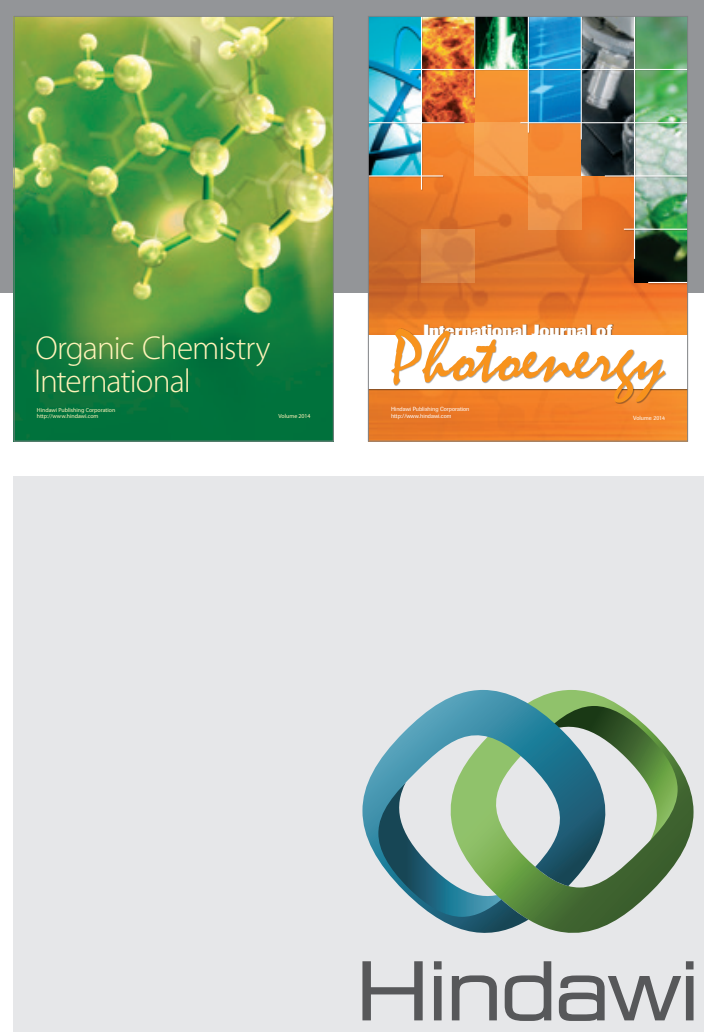

Submit your manuscripts at

http://www.hindawi.com

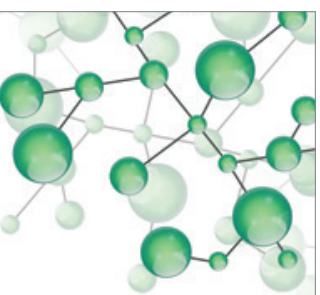

International Journal of

Inorganic Chemistry

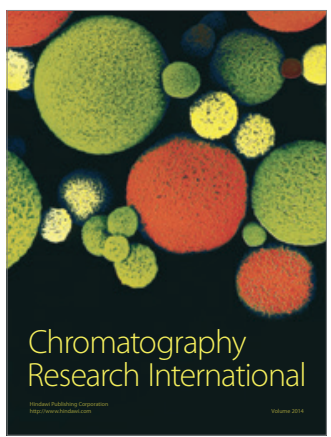

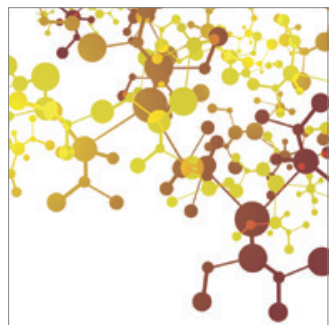

Applied Chemistry
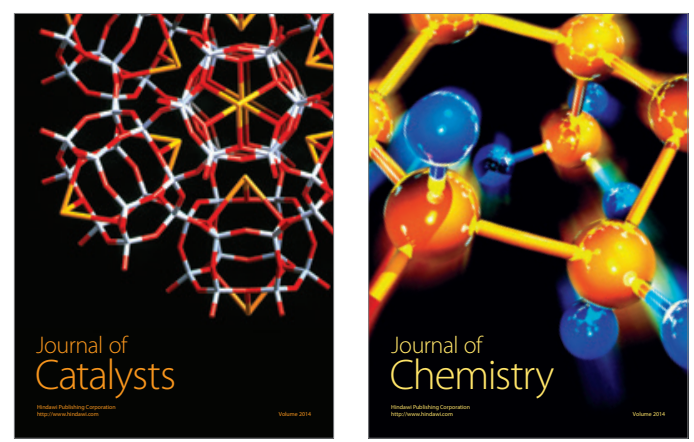
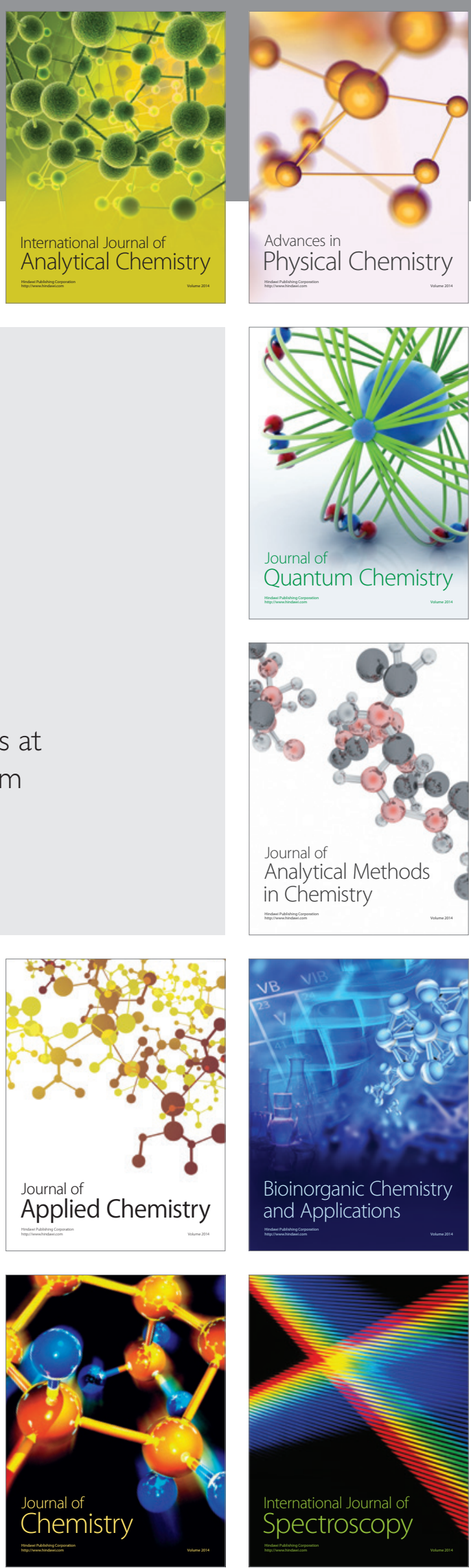